\title{
Essai de quantification des termes du bilan hydrique des étangs du système Vaccarès (Camargue, France)
}

\author{
P. Heurteaux ${ }^{1}$
}

Mots clés : Camargue, Vaccarès, étangs salés, bilans hydriques, écosystèmes lagunaires perturbés.

Les termes du bilan hydrique des étangs du système Vaccarès sont analysés. Les pluies et l'apport d'eau de drainage agricole constituent l'essentiel de l'actif du bilan auquel s'ajoutent éventuellement les entrées d'eau de mer, volontaires ou accidentelles, les entrées d'eau douce volontaires et les apports des eaux souterraines. L'évaporation et l'évacuation en mer du trop-plein des étangs constituent l'essentiel des sorties d'eau.

Malgré la rareté de l'information, la quantification des termes du bilan est tentée en première approche. Pour une période donnée, la prépondérance des entrées d'eau sur les sorties, ou inversement, se traduit par un stockage ou un destockage et par des variations du niveau et de la salinité des étangs.

\section{An attempt to quantify the terms of the hydrological balance for the lagoons of the Vaccares system}

Keywords : Camargue, Vaccarès, saline lagoon, water-balance, perturbed lagoon ecosystem.

The terms of the hydrological balance for the lagoons of the Vaccarès system are analysed. Rainfall and inflow from agricultural drainage constitute almost all of the inputs to the balance, possibly together with intentional or accidental inflows of sea water, intentional inputs of fresh water and groundwater inputs. Evaporation and outflows to the sea at high water levels in the lagoons are the main water outputs.

Despite the paucity of information, a preliminary attempt at quantifying the terms is attempted. For any given period, inputs of waters may dominate over outputs, or vice versa, leading either to storage or decrease in stored volume and to change in level and salinity in the lagoons.

Les étangs du système Vaccarès ont des niveaux et des salinités variables très largement influencés par les activités humaines. Les niveaux contrôlent à distance plusieurs facteurs dont dépend le fonctionnement écologique de toute la Camargue. Ils interviennent sur l'efficacité du drainage superficiel, sur la profondeur des nappes d'eaux souterraines laquelle, à son tour, agit sur la dynamique de l'eau dans la zone d'aération du sol et sur l'importance de la salinisation des terrains. Mes recherches sur la dynamique de l'eau et du sel des étangs centraux de la Grande Camargue (Heurteaux 1988 ; Vaquer \& Heurteaux 1989, Heurteaux 1992) m'ont amené à essayer de quantifier les termes de leur bilan hydrique, entreprise quelque peu hasardeuse du fait de la pauvreté de l'information, mais ce travail de défrichement indispensable comme préalable à une informatisation du fonctionnement hydraulique du Delta du Rhône.

\section{Aperçu géomorphologique et hydro- logique du bassin versant du système Vaccarès}

L'Ile de Camargue couvre entre les deux bras du Rhône une superficie de quelque $800 \mathrm{~km}^{2}$. Elle a la forme d'un amphithéâtre dont la partie basse, adossée à la mer est occupée par les étangs du système Vaccarès (Fig. 1). En périphérie de ces étangs, les terrains qui les enserrent en fer à cheval ont, depuis les rives du Rhône, une déclivité très faible, de l'ordre de $0,3 \%$, mais suffisante pour assurer un drainage naturel centripète. Le bassin versant total du système couvre quelque 470 $\mathrm{km}^{2}$. Les plans d'eau occupent une vaste superficie mais ils sont peu profonds et leur volume est modeste (encart). Des passes assurent l'intercommunication entre les différents étangs et baisses qui constituent une unité hydraulique, mais le fonctionnement hydraulique du Vaccarès proprement dit est très différent de celui des Etangs inférieurs.

1. C.N.R.S., Laboratoire d'Hydrobiologie, Université de Provence, 13331 Marseille Cedex 03. 


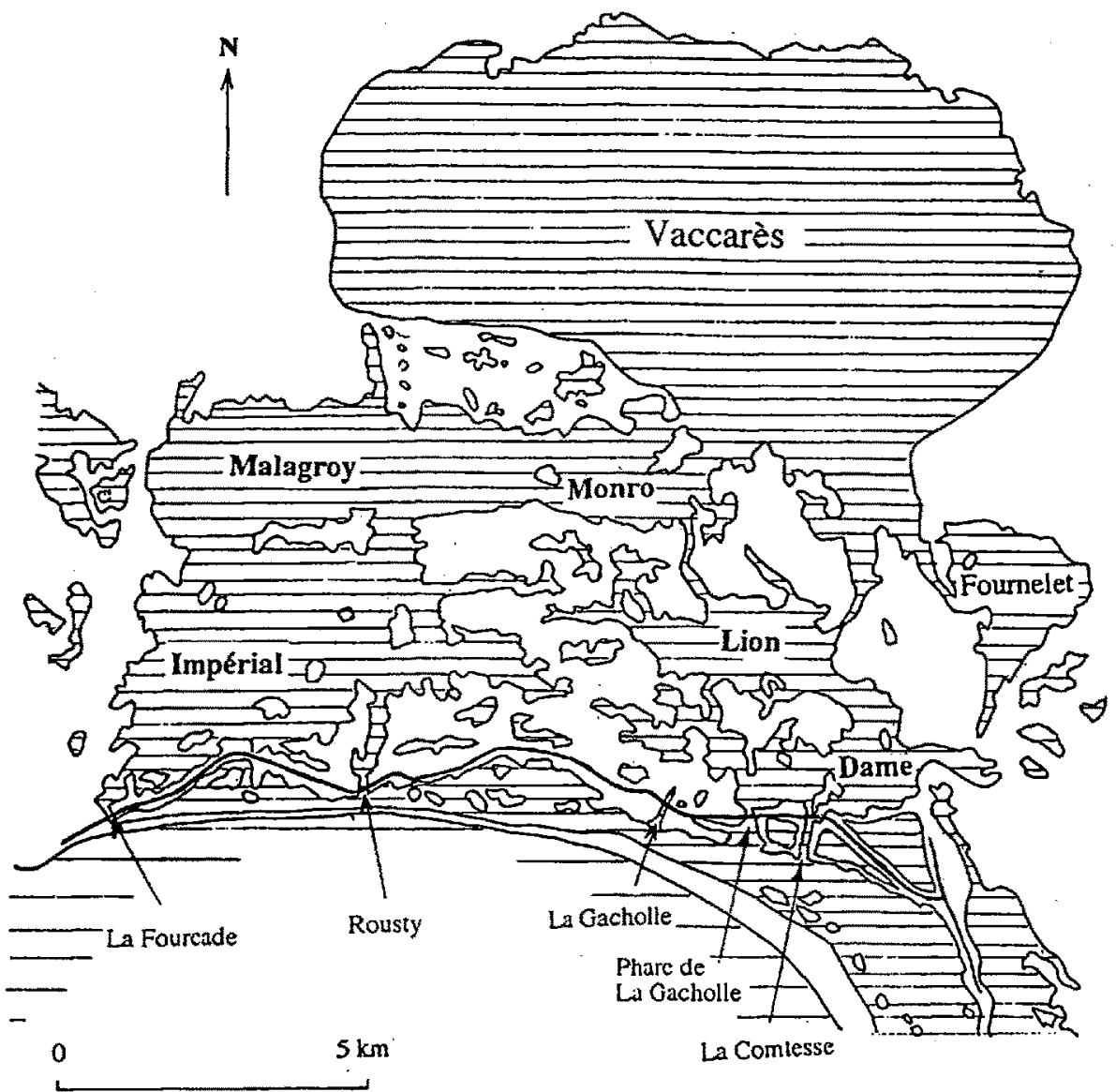

Fig. 1. Les étangs du système Vaccarès.

Au sud des Etangs inférieurs, la Digue à la Mer (trait épais) et ses vannages appelés pertuis. Fig. 1. The lagoons of the Vaccarès system.

To the South the lower lagoons, the sea wall (thick line) and its sluices known as "pertuis».

\begin{tabular}{|c|c|c|c|}
\hline & $\begin{array}{l}\text { Etang du } \\
\text { Vaccares }\end{array}$ & $\begin{array}{l}\text { Etangs } \\
\text { inférieurs }\end{array}$ & $\begin{array}{c}\text { total } \\
\text { systeme Vaccarès }\end{array}$ \\
\hline \multicolumn{4}{|l|}{ Superficie du bassin versant } \\
\hline - les étangs & $67 \mathrm{~km} 2$ & $45 \mathrm{~km} 2$ & $112 \mathrm{~km} 2$ \\
\hline - les terres potentiellement drainables & $317 \mathrm{~km} 2$ & $40 \mathrm{~km} 2$ & $357 \mathrm{~km} 2$ \\
\hline- total & $384 \mathrm{~km} 2$ & $85 \mathrm{~km} 2$ & $469 \mathrm{~km} 2$ \\
\hline Terres effectivement drainées & $130 \mathrm{~km} 2$ & $25 \mathrm{~km} 2$ & $155 \mathrm{~km} 2$ \\
\hline \multicolumn{4}{|l|}{ Profondeur maximale des étangs } \\
\hline a la cote zéro NGF & $2,1 \mathrm{~m}$ & $0,8 \mathrm{~m}$ & \\
\hline \multicolumn{4}{|l|}{ Profondeur moyenne des étangs } \\
\hline a la cote zéro NGF & $1,4 \mathrm{~m}$ & $0,4 \mathrm{~m}$ & \\
\hline Volume des étangs à la cote zéro NGF & $93 \mathrm{Mm} 3$ & $21 \mathrm{Mm3}$ & $114 \mathrm{Mm} 3$ \\
\hline
\end{tabular}

Le bassin versant du système Vaccarès et principales caractéristiques des étangs. The bassin of the Vaccares system and main characteristics of the lagoons. 
En effet, l'étang du Vaccarès peut recueillir les eaux de $86 \%$ du bassin versant terrestre total du système comprenant beaucoup de terres hautes bien drainées par un réseau de drains efficaces, alors que les Etangs inférieurs sont plus ou moins en relation avec une quarantaine de $\mathrm{km}^{2}$ de terrains bas amphibies et halomorphes (sansouires), mal drainés.
Le bassin versant terrestre de l'étang du Vaccarès comprend la plus grande partie des terres agricoles camarguaises, notamment les rizières. Il est divisé en bassins culturaux (Ministère de l'agriculture, 1970, 1975, 1983) (Fig. 2) :

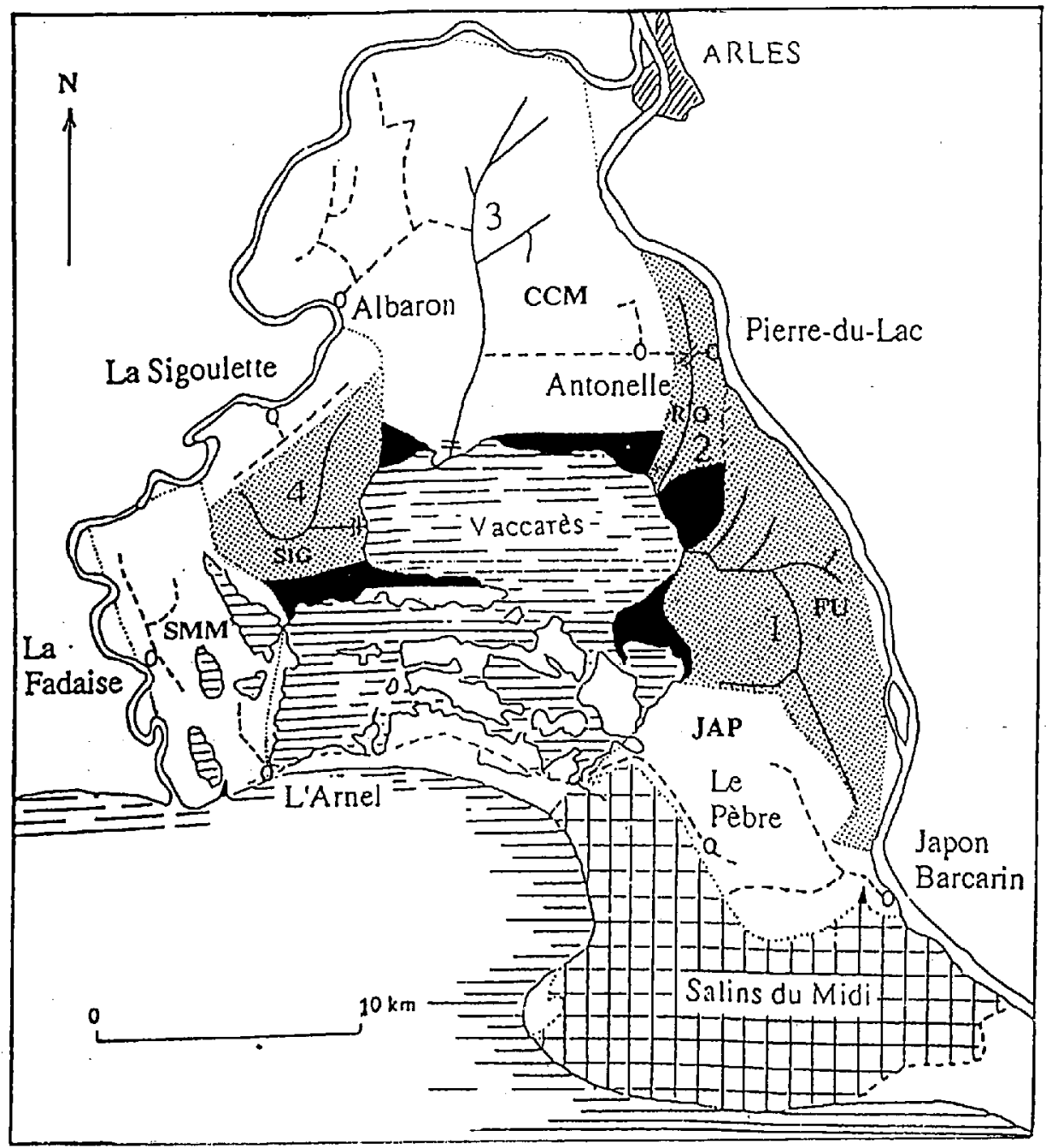

Fig. 2. Les bassins culturaux et le réseau de drainage de la Camargue (d'après DDAF 1975). - En trait pointillé, les limites des bassins : JAP, bassin du Japon ; FU, bassin de Fumemorte ; RO, bassin de Roquemaure ; CCM, bassin de Corrège et Camargue major ; SIG, bassin de la Sigoulette ; SMM, bassin des Saintes-Maries-de-la-Mer. - En noir (terres riveraines) et en pointillés, partie du bassin versant terrestre effectivement drainée au Vaccarès. - En trait plein, les 4 grands canaux tributaires du Vaccarès : Fumemorte (1), Roquemaure (2), Rousty (3) et Sigoulette (4). - Le réseau d'assainissement du plan de 1955, ses canaux (trait tireté) et ses stations de rejet des eaux au Rhône ou à la mer (o).

Fig. 2. The agricultural drainage basins and the drainage network of the Camargue (from DDAF 1975). - The boundaries of the basins : JAP, Japan basin ; FU, Fumemorte basin ; RO, Roquemaure basin ; CCM, Corrège and Camargue major basin ; SIG, Sigoulette basin ; SMM, Saintes-Maries-de-la-Mer basin. - In black (waterside lands) and in dotted area ; part of the drainage basin effectively drained to the Vaccarès. - ASH continuous lines; the 4 main canals draining into the Vaccarès : Fumemorte (1) ; Roquemaure (2) ; Rousty (3) ; La Sigoulette (4). - The drainage network of the 1955 plan, its canals (dashed lines) and its stations for pumping water back to the Rhône or sea $(0)$. 
- à l'est, les bassins de Fumemorte $\left(66 \mathrm{~km}^{2}\right)$ et de Roquemaure $\left(14 \mathrm{~km}^{2}\right)$ sont drainés par les canaux de mêmes noms ;

- au nord, le bassin de Corrège et Camargue major $\left(178 \mathrm{~km}^{2}\right)$ est drainé naturellement par le canal de Rousty ;

- à l'ouest, le bassin de Sigoulette $\left(35 \mathrm{~km}^{2}\right)$ est drainé naturellement par le canal de même nom.

L'étang draine en outre environ $25 \mathrm{~km}^{2}$ de terrains riverains.

Conformément au plan d'assainissement mis en place dès 1955 , les bassins nord et ouest sont équipés de stations de pompage permettant de rejeter au Rhône, tout ou partie des eaux de drainage agricoles et pluviales (Fig. 2). Les canaux de Rousty et de la Sigoulette sont barrés à leur embouchure par des vannages et peuvent être isolés du Vaccarès. Le bassin nord est entièrement poldérisable. Le bassin ouest comprend les terres hautes agricoles, proches du Rhône, assainies par repompages au fleuve et quelque $25 \mathrm{~km}^{2}$ de terres basses marécageuses non cultivées drainées au Vaccarès par le canal de la Sigoulette. Un canal de décharge relie les deux compartiments du bassin et permet, suivant les circonstances, de moduler les rejets au fleuve ou à l'étang. Les bassins orientaux ne sont pas pourvus d'installations d'assainissement au Rhône. Les canaux de Fumemorte et Roquemaure déversent par gravité au Vaccarès l'intégralité des eaux et drainage qu'ils recueillent.

Pour des raisons de rentabilité agricole, depuis une quinzaine d'années, sauf pour des conditions climatiques exceptionnelles, la plus grande partie des terres pouvant être assainies par repompages au Rhône fonctionnent en autonomie. Sur les $317 \mathrm{~km}^{2}$ du bassin versant terrestre du Vaccarès, seuls 130 $\mathrm{km}^{2}$ sont drainés par gravité à l'étang. Toutefois, une vanne de décharge sur le canal de Roquemaure permet d'écouler par gravité au Vaccarès une partie non négligeable des colatures du bassin nord.

Contrairement au Vaccarès, les Etangs inférieurs ne drainent pas de terres agricoles. Ils sont séparés les uns des autres par des sansouires basses, peu déclives, aux limites floues (cotes comprises entre $-0,10$ et $+0,20 \mathrm{~m} \mathrm{NGF}$ ), exondées l'été, inondées l'hiver, annexées partiellement ou totalement aux étangs par hautes eaux. Environ $20 \mathrm{~km}^{2}$ de ces terres interviennent directement dans le fonctionnement hydraulique des étangs.
Le bilan hydrique du sytème Vaccarès est régi par plusieurs variables en entrées et en sorties. Malgré l'intervention d'aménagement anthropiques (irrigation, endiguements), l'action antagonistes des pluies et de l'évaporation joue un rôle dominant. Suivant l'importance relative des différents postes du bilan, les étangs du système Vaccarès ont des niveaux variables qui évoluent à l'intérieur de limites extrêmes qui, depuis le développement de la riziculture, sont $-0,45$ et $+0,45$ m NGF. Pour un pas de temps donné, les entrées et les sorties sont rarement équilibrées, en fin d'exercices il y a le plus souvent un solde positif ou négatif qui se traduit par le stockage ou le destockage d'un certain volume d'eau. Les entrées et les sorties concernent des eaux plus ou moins salées, ce qui entraîne des variations temporelles et spatiales de la salinité des étangs. Ces variations de salinités et leurs causes ont été étudiées précédemment (Heurteaux, 1992). Je me propose, dans le présent travail, de faire une analyse qualitative et quantitative du bilan volumique des étangs.

\section{Les termes du bilan hydrique du système Vaccarès}

\subsection{Les entrées d'eau}

\subsubsection{Les précipitations}

Les relevés de Météofrance font état de six stations pluviométriques dont deux concernent la haute Camargue (Arles-ville et mas de Rousty), deux la moyenne Camargue (Tour du Valat et le Sambuc) et deux la basse Camargue (Salin de Giraud et Saintes-Maries-de-la-Mer). Ces stations révèlent une forte irrégularité des pluies dans le temps et dans l'espace. En moyenne, il existe un gradient croissant des précipitations du sud au nord. Le module pluviométrique annuel de la Moyenne Camargue est de $593 \mathrm{~mm}$ (48 ans) avec un écart type de 170 et des extrêmes de $250 \mathrm{~mm}$ (1989) à $955 \mathrm{~mm}$ (1969).

L'apport d'eau pluviale aux étangs provient de l'interception directe des plans d'eau et du ruissellement sur le bassin versant terrestre. Celui-ci est sujet à de fortes variations temporelles en fonction de la hauteur et de la durée des pluies, de la densité du réseau de drains et de la capacité d'emmagasinement du bassin versant terrestre au moment où tombent les pluies, capacité d'emmagasinement qui dépend du niveau des eaux souterraines et de l'état hydrique de la zone d'aération du sol. 
Aux variations temporelles des apports d'eau pluviale s'ajoutent des variations spatiales. Ainsi, le rendement d'une même pluie est, en général, meilleur pour le Vaccarès que pour les Etangs inférieurs, ceux-ci ayant un bassin versant terrestre relativement plus réduit.

L'eau de ruissellement pluvial recueillie par le Vaccarès est légèrement minéralisée $(0,8 \mathrm{~g} / 1$ en moyenne d'après Godin (1990). Elle peut être plus salée dans la zone des Etangs inférieurs.

\subsubsection{Le drainage agricole}

Pendant toute la première moitié du $\mathrm{XX}^{\mathrm{e}}$ siècle, la submersion automnale de vignoble pour lutter contre le Phylloxéra a fourni l'essentiel des apports agricoles au Vaccarès. Le vignoble camarguais qui comptait pour tout le delta 8600 ha en 1933 et encore quelque 3000 ha entre 1960 et 1980 a pratiquement disparu au cours de la décennie 1980. En 1991, il ne restait que 385 ha de vignes entre les deux bras du Rhône (Parc naturel régional de Camargue 1992). Depuis 1950, la part la plus importante des retours d'irrigation qui s'écoulent au Vaccarès est d'origine rizicole. Les cultures irriguées classiques (maïs, tournesol, maraîchages, prairies etc.) n'exportent pas d'eau de drainage.

Les colatures agricoles sont légèrement minéralisées.

\subsubsection{Les introductions d'eau de mer par la digue littorale (" Digue à la mer »)}

La Digue à la mer est percée de vannages destinés en principe à l'écoulement en mer du trop-plein hivernal des étangs, mais, de tout temps, les vannes ont souvent fait l'objet de manipulations illicites pour favoriser l'entrée des poissons marins dans les étangs. A ces introductions volontaires d'eau de mer s'ajoutent parfois des intrusions involontaires dues à des fuites ou à des ruptures de digue. Les entrées d'eau de mer, accidentelles ou intentionnelles, particulièrement importantes de 1978 à 1988 , ont pesé très lourd sur les bilans salins.

\subsubsection{Apport d'eau souterraine dans la zone des Etangs inférieurs}

Sur la plus grande partie de sa surface, le fond argilo-limoneux du Vaccarès a une très faible conductivité hydraulique qui minimise les éventuels échanges d'eau entre l'étang et son sous-sol et les rendent négligeables à l'échelle d'un bilan annuel.
Par contre, les fonds à tendance sableuse de la partie sud de l'étang, dans le prolongement de la presqu'île de Mornès, et de toute la zone des Etangs inférieurs, sont perméables et autorisent, sous certaines conditions, l'apport d'eaux souterraines aux étangs suivant un mécanisme décrit par ailleurs. De même, sous certaines conditions, il peut y avoir apport d'eaux souterraines aux sansouires bordant les étangs lorsqu'elles sont inondées (Heurteaux 1988 ; Heurteaux 1993). Globalement, les apports d'eaux souterraines sont peu importants en volume et leur intervention sur les bilans hydriques restent modeste, par contre, c'est une source de sels non négligeable pour le système. En effet, la moitié sud de la Camargue a été conquise sur la mer, les sédiments finiholocènes qui la constituent ont été déposés en milieu lagunaire et les eaux souterraines qui imprègnent ses sédiments sont salées, voire sursalées, même sous les étangs ( $>50 \mathrm{~g} / \mathrm{l})$.

\subsubsection{Les apports anthropiques incontrôlables}

Pour la seule Grande Camargue, quelque 3000 ha de marais sont aménagés et maintenus en eau en été pour la chasse au gibier d'eau. Ces marais sont raccordés au réseau de drainage et si, en principe, ils ne sont pas drainés, des fuites et des surverses incontrôlables sont possibles.

D'autre part, les basses terres incultes du bassin des Saintes-Maries-de-la-Mer sont assainies par une station d'exhaure qui rejette l'eau en partie en mer et en partie; non chiffrée, dans l'étang de l'Impérial. En appoint à ce système d'assainissement principal du bassin, des canaux relient aux étangs de Malagroy et de l'Impérial une zone marécageuse de $15 \mathrm{~km}^{2}$ comprenant les étangs de Ginès et de Consécanière. Cette zone est potentiellement drainable par gravité, mais le fonctionnement des vannages barrant les canaux de communication n'est pas codifié et les apports éventuels au système Vaccarès ne peuvent pas être quantifiés. Des apports intentionnels non négligeables ont souvent lieu en été lorsque les étangs atteignent des salinités incompatibles avec la pêche.

\subsection{Les sorties d'eau}

\subsubsection{L'évaporation des plans d'eau}

Les mesures que j'ai faites en bac Colorado en Moyenne Camargue de 1962 à 1978 indiquent une évaporation moyenne de l'eau libre de $1180 \mathrm{~mm}$ 
(de 1 à 1,3 m) avec un écart type de 78. L'évaporation mensuelle calculée par la formule de Penman est bien corrélée avec les mesures en bac (Heurteaux 1990) et peut être utilisée pour l'établissement des bilans. La salinité de l'eau doit être prise en compte dans les calculs puisque l'évaporation diminue de $1 \%$ quand la salinité s'élève de $1 \%$.

\subsubsection{L'évacuation du trop-plein des étangs par les vannes de la digue littorale ("Digue à la Mer ")}

La Digue à la mer a été conçue pour empêcher les intrusions marines dans les basses terres lors des tempêtes tout en permettant d'évacuer le trop-plein hivernal des étangs. Pour cela; le corps de la digue a été percé de vannages appelés pertuis. A l'origine, il y avait deux pertuis, totalisant $34 \mathrm{~m}$ d'ouverture, aménagés, l'un, à l'est, au droit du systène d'étangs Lion-Dame-Marteau (pertuis de la Comtesse), l'autre à l'ouest au droit du système Monro-MalagroyImpérial (pertuis de Rousty). Les besoinis de la viticulture puis de la riziculture ont conduit à rendre plus performant le système d'évacuation pour faire face à l'apport massif d'eau de drainage agricole, d'abord en rationalisant son exploitation, puis en perçant quatre nouveaux vannages, portant l'ouverture à $86 \mathrm{~m}$. Il s'agit d'est en ouest des pertuis de la nouvelle Comtesse, du phare de la Gacholle, de la Gacholle et de la Fourcade. Avec le déclin de la riziculture, les ouvrages mal entretenus ont perdu de leur efficacité. Aujourd'hui, le pertuis de la Fourcade en contact direct avec la mer assurc seul l'évacuation du trop-plein des étangs. Ces dếparts d'eau s'accompagnent pour le système d'étangs de pertes de sel dont l'importance est fonction de la salinité de l'étang de l'Impériai.

\subsubsection{Les pertes des Elangs inférieurs par infiltration}

Les étangs peuvent occasionnellement alimenter la nappe sous-jacente. Les volumes d'eau et les pertes de sels ainsi mis en jeu sont vraisemblablement peu importants.

\section{Essai de quantification des termes clima- tiques du bilan hydrique du système Vaccarès}

\subsection{Les précipitations}

\subsubsection{Cas du Vaccarès}

Dans l'état actuel de nos connaissances, il est difficile de quantifier la part de la pluie incidente recueillie par l'étang par apport direct et par ruis- sellement. Le calcul de l'apport pluvial direct n'offre pas de difficulté, les différences de niveaux de l'étang affectent relativement peu la surface du Vaccarès qui, entre ses cotes extrêmes, reste comprise entre 66 et $68 \mathrm{~km}^{2}$. Par contre, le calcul des cœefficients de ruissellement des pluies pose problème. Ces cœfficients n'ont en effet jamais fait l'objet de mesures directes précises. J'ai pu toutefois mettre en relation la hauteur des pluies et l'élévation du niveau du système d'étangs pour de rares périodes où l'intervention des autres facteurs agissant sur les niveaux est négligeable ou quantifiable. Ces observations montrent que l'importance du ruissellement pluvial est largement tributaire de l'état hydrique du sol et de l'intensité des pluies. Ainsi, en période sèche, l'apport pluvial au Vaccarès par ruissellement peut varier de 0 à $55 \%$ de la pluie incidente suivant qu'il s'agit de faibles ondées ou d'orages violents ; en période pluvieuse, le bassin versant terrestre cède à l'étang 50 à $95 \%$ de la pluie incidente suivant le degré de saturation des terrains.

En première approximation, pour quantifier l'apport par ruissellement pluvial, il semble raisonnable de retenir trois états hydriques du sol et pour chacun d'eux les cœfficients de ruissellement suivants : 0,0 à 0,3 pour les sols secs (s), 0,6 à 0,8 pour les sols humides (h) et 0,4 à 0,5 pour un état hydrique intermédiaire (i). Pour chaque épisode pluvieux d'un mois donné, la part du ruissellement est déterminée au coup par coup par un examen détaillé et critique des tableaux climatologiques de Météofrance. Les hauteurs de pluie prise en compte pour un bassin versant terrestre réduit à $130 \mathrm{~km}^{2}$, sont celles relevée à la station climatologique de la Tour du Valat éventuellement corrigées des données du Sambuc.

L'approximation des cœfficients de ruissellement ajouté au fait que l'apport par ruissellement d'un épisode pluvieux peut s'étaler dans le temps n'autorise les calculs que sur des pas de temps assez longs, le mois semble un minimum.

\subsubsection{Cas des Etangs inférieurs}

Contrairement au Vaccarès, l'apport pluvial direct aux étangs est prépondérant, mais la superficie des Etangs inférieurs varie beaucoup en fonction des niveaux. Ils découvrent des plages par basses eaux ( $\mathrm{n}<-0,20 \mathrm{~m} \mathrm{NGF}$ ) et débordent sur les sansouires par hautes eaux jusqu'à les annexer totalement pour des niveaux supérieurs à $+0,20 \mathrm{~m}$ NGF. Le sol des plages conserve en permanence un taux 
d'humidité élevé ce qui n'est guère favorable à l'infiltration des pluies, par contre leur déclivité est favorable au ruissellement centripète des pluies relativement importantes. Les sansouires, quant à elles, sont mal drainées et la moitié seulement des quelque $40 \mathrm{~km}^{2}$ constituant le bassin versant terrestre intervient directement dans le bilan hydrique des étangs. Pour calculer le volume des pluies efficaces, à partir de ces données qualitatives et de la connaịssance des lieux, pour les différents niveaux et pour les trois états hydriques du sol déjà mentionnés, il semble raisonnable d'appliquer aux pluies incidentes mensuelles exprimées en $\mathrm{mm}$, les coefficients indiqués sur la Tableau $1 \mathrm{~A}$.

Tableau 1. Coefficients à appliquer aux hauteurs de pluie (A) ou d'évaporation (B) en mm pour obtenir, en fonction de la cote NGF du Vaccarès lue à l'échelle de Fumemorte (nFu NGF) : A, l'apport pluvial aux Etangs inférieurs du Vaccarès, en millions de ${ }^{3}$, suivant l'état hydrique des terrains ; $B$, le volume évaporé en millions de $\mathrm{m}^{3}$ au niveau du Vaccarès et des Etangs inférieurs.

Table 1. Factors to be applied to rainfall quantities in mm to obtain, depending on the level of the Vaccarès above sea level (NGF) as measured at the Fumemorte (nFu NGF) : A, the rainfall inputs to the lower lagoons (Etangs inférieurs) en millions $\mathrm{m}^{3}$, depending on hydrological state of land; $\mathrm{B}$, the volume evaporated from the Vaccarès and the lower lagoons in millions $\mathrm{m}^{3}$.

$\mathbf{A}$

\begin{tabular}{|c|c|c|c|}
\hline n Fu NGF & & $\begin{array}{l}\text { Etat hydrique } \\
\text { des terrains }\end{array}$ & \\
\hline & sec & intermédiaire & humide \\
\hline$>+0,30 \mathrm{~m}$ & 0,065 & 0,065 & 0,065 \\
\hline$+0,20 \mathrm{~m}$ & 0,060 & 0,060 & 0,060 \\
\hline$+0,10 \mathrm{~m}$ & 0,050 & 0,050 & 0,055 \\
\hline zéro & 0,045 & 0,045 & 0,050 \\
\hline$-0,10 \mathrm{~m}$ & 0,045 & 0,045 & 0,050 \\
\hline$-0,20 \mathrm{~m}$ & 0,045 & 0,045 & 0,050 \\
\hline$-0,30 \mathrm{~m}$ & 0,043 & 0,045 & 0,050 \\
\hline$-0,40 \mathrm{~m}$ & 0,037 & 0,040 & 0,042 \\
\hline$-0,50 \mathrm{~m}$ & 0,030 & 0,035 & 0,040 \\
\hline
\end{tabular}

B

\begin{tabular}{ccc}
\hline $\begin{array}{c}\text { Etang du } \\
\text { Vaccarès }\end{array}$ & $\begin{array}{c}\text { Etangs } \\
\text { inférieurs }\end{array}$ & n Fu NGF \\
\hline & & \\
$\mathbf{0 , 0 6 8}$ & 0,065 & $>+0,30 \mathrm{~m}$ \\
$\mathbf{0 , 0 6 7}$ & 0,060 & $+0,20 \mathrm{~m}$ \\
$\mathbf{0 , 0 6 7}$ & 0,050 & $+0,10 \mathrm{~m}$ \\
$\mathbf{0 , 0 6 6}$ & $\mathbf{0 , 0 4 5}$ & zéro \\
$\mathbf{0 , 0 6 6}$ & $\mathbf{0 , 0 4 5}$ & $-0,10 \mathrm{~m}$ \\
$\mathbf{0 , 0 6 6}$ & $\mathbf{0 , 0 4 3}$ & $-0,20 \mathrm{~m}$ \\
$\mathbf{0 , 0 6 6}$ & $\mathbf{0 , 0 3 7}$ & $-0,30 \mathrm{~m}$ \\
$\mathbf{0 , 0 6 6}$ & $\mathbf{0 , 0 2 2}$ & $-0,40 \mathrm{~m}$ \\
$\mathbf{0 , 0 6 6}$ & $\mathbf{0 , 0 1 0}$ & $-0,50 \mathrm{~m}$ \\
\hline
\end{tabular}

Pour tenir compte du gradient NS des pluies, les. hauteurs moyennes des précipitations prises en compte pour les calculs sont celles des stations de la Tour du Valat (TV), de Salin de Giraud (SG) et des Saintes-Maries-de-la-Mer (SMM) modulées de la façon suivante $: \frac{1}{4}(2 \mathrm{TV}+\mathrm{SG}+\mathrm{SMM})$

\subsubsection{Ensemble du système}

L'apport total d'eau pluviale aux étangs a été calculé pour 110 mois en observant les règles ci-dessus. Malgré l'influence de l'état hydrique du bassin versant, cet apport est bien corrélé avec la hauteur des précipitations sauf en période très sèche lorsque les Etangs inférieurs ont tendance à s'assécher. En première approximation, on peut calculer les volumes d'eau en millions de $\mathrm{m}^{3}$ ápportés aux étangs par les pluies exprimées en millimètres en appliquant la relation $\mathrm{V}=$ 0,2.P (Fig. 3).

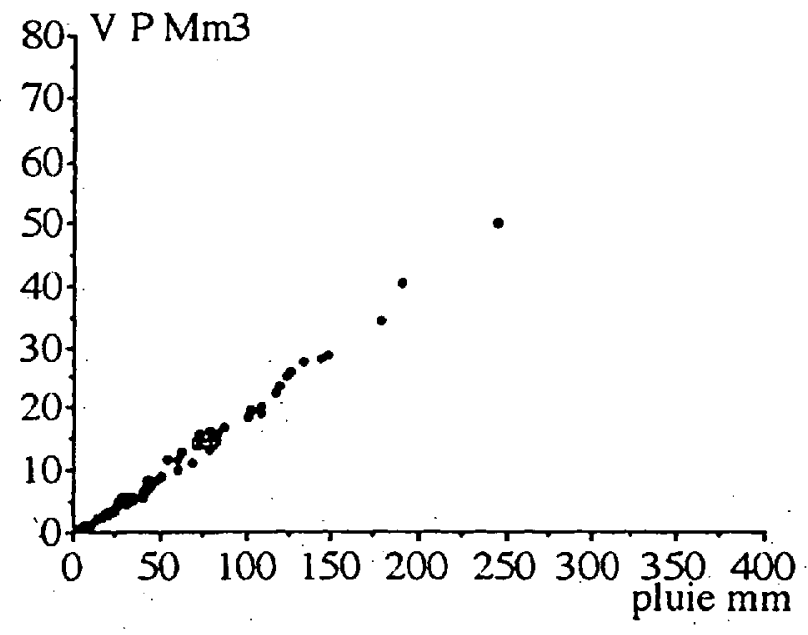

Fig. 3. Relation entre l'apport pluvial mensuel aux étangs du système Vaccarès (VP en millions de $\mathrm{m}^{3}$ ) et la hauteur des précipitations mensuelles ( $\mathrm{n}=110$ ).

Fig. 3. Relationship between the inflow of rain water into the lagoon of the Vaccarès system (VP in millions of cubic metres) and the amount of monthly rainfall $(n=110)$. 


\subsection{L'évaporation}

Pour quantifier les pertes par évaporation pendant un pas de temps donné, l'évaporation mensuelle calculée par la formule de Penman, éventuellement minorée pour tenir compte de la salinité de l'eau, est rapportée à la surface moyenne des étangs pendant la période considérée. On obtient le volume des pertes par évaporation en millions de $\mathrm{m}^{3}$ en fonction du niveau des étangs en affectant l'évaporation exprimée en mm d'eau par les coefficients indiqués sur le tableau $1 \mathrm{~B}$.

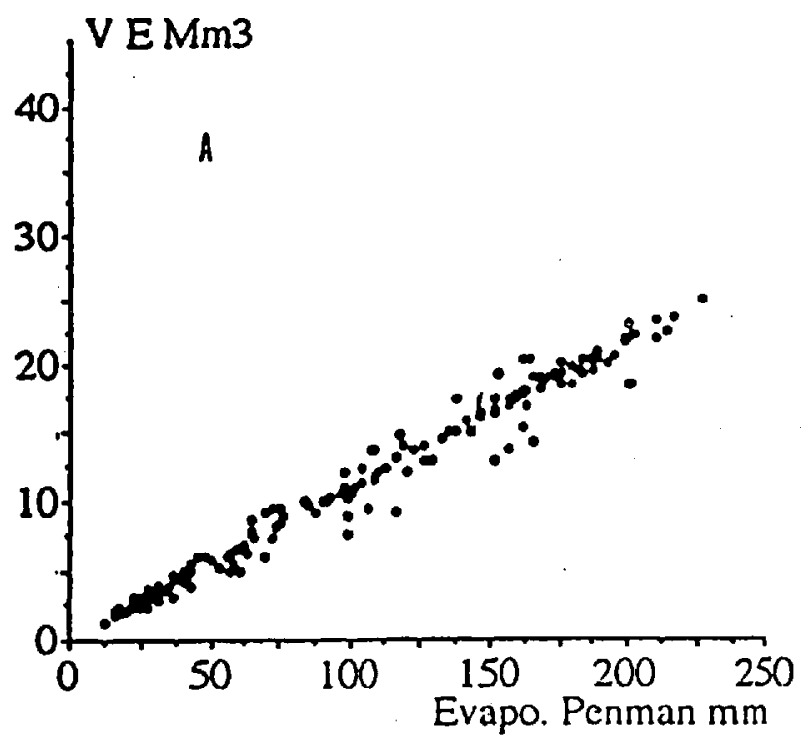

L'intervention de la salinité et, surtout, de la dimension de la surface évaporante (Fig. 4 B), peut induire, pour une même valeur de l'évaporation Penman, d'importantes variations du volume évaporé mensuel. Il est donc préférable de calculer celuici au coup par coup, mais en première approche, la relation $V=0,107 \mathrm{EPEN}$, où EPEN est l'évaporation Penman en mm, donne une assez bonne approximation du volume en millions de $\mathrm{m}^{3}$ soustrait par évaporation (Fig. 4 A).

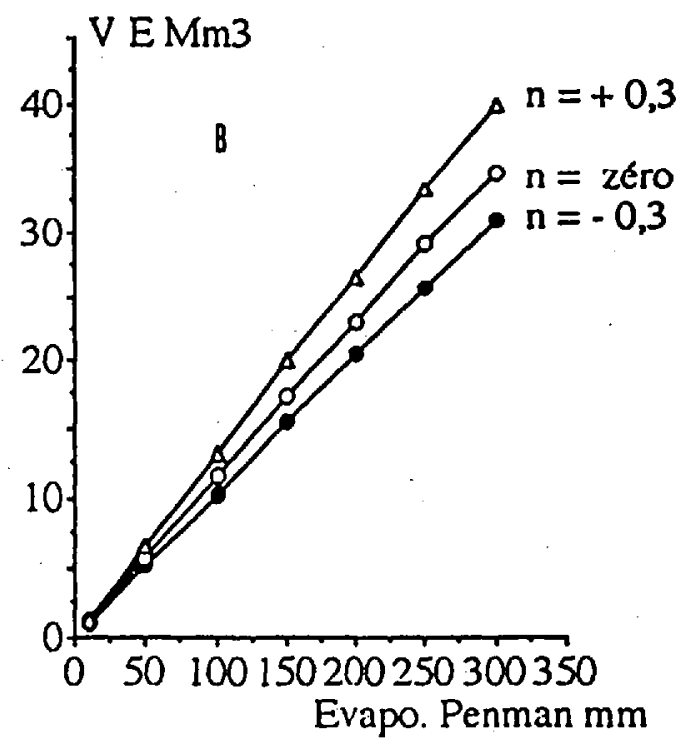

Fig. 4. Evolution du volume mensuel soustrait aux étangs du système Vaccarès par évaporation (VE en millions de $\mathrm{m}^{3}$ ) en fonction de l'évaporation mensuelle et de la surface évaporatoire des plans d'eau en fonction de leurs cotes NGF (A, $n=179)$. Pour d'égales valeurs de l'évaporation, intervention de la surface évaporatoire sur le volume évaporé pour les trois cotes $-0,30 \mathrm{~m}$, zéro et $+0,30 \mathrm{~m}$ NGF (B).

Fig. 4. Changes in the monthly volume evaporating from the lagoons of the Vaccares system (VE in millions of cubic metres) according to the Penman monthly evaporation and the altitude of the lagoons above sea level $(A, n=179)$. For the same values of evaporation, influence of the water surface area on the importance of evaporation rate for three levels above the sea : $-0,30 \mathrm{~m}$, sea level and $+0.30 \mathrm{~m}(\mathrm{~B})$.

\section{Essai de quantification des termes anthro- piques du bilan hydrique du système Vaccarès}

\subsection{Les entrées d'eau du drainage agricole}

- Eaux de drainage d'angime viticole - L'apport ofomnal d'eau de drainage d'origine viticole au
Vaccarès est à citer pour mémoire. Il ne représente plus qu' 1 à 2 millions de $\mathrm{m}^{3}$ par an aujourd'hui alors qu'il était encore de 15 millions de $\mathrm{m}^{3}$ en 1980 et qu'il a atteint 40 à 50 millions de $\mathrm{m}^{3}$ par an au cours de la décennie 1930 (Hetrrteaux 1988). 
- Eaux de drainage d'origine rizicole - Le volume des colatures rizicoles épanchées au Vaccarès dépend de la surface cultivée en rizières et du mode d'irrigation pratiqué. L'eau distribuée aux rizières est évapotranspirée par le système rizière-riz $\left(10000 \mathrm{~m}^{3} / \mathrm{ha}\right.$ par an en moyenne), s'infiltre (6000 $\mathrm{m}^{3} /$ ha en moyenne) ou alimente le drainage superficiel. Le volume directement exporté dans l'environnement varie de 1 à 5 (de 5000 à $25000 \mathrm{~m}^{3} / \mathrm{ha} / \mathrm{an}$ ) suivant le mode d'irrigation des rizières (irrigation classique ou " à la surverse »). L'irrigation « à la surverse ", appellation évocatrice d'une surconsommation d'eau par rapport aux besoins réels, fut pratiquée généreusement au cours de la décennie 1960 , elle est plus modérée aujourd'hui et en moyenne, le drainage superficiel ne dépasse pas $12000 \mathrm{~m}^{3} / \mathrm{ha}$ par an. Cette eau est en partie utilisée pour le maintien en eau des marais de chasse, le reste est éliminé par repompages au Rhône ou par écoulement gravitaire au Vaccarès. Le Vaccarès collecte des eaux agricoles des bassins du Fumemorte et Roquemaure à raison d'environ $10000 \mathrm{~m}^{3}$ par hectare de rizière. L'apport des propriétés riveraines est estimé à $12000 \mathrm{~m}^{3} /$ ha riz. Le canal de Roquemaure collecte en outre une partie des eaux de drainage du bassin nord ( 8 à 10 millions de $\mathrm{m}^{3}$ par an dont les deux tiers environ en été). Donc, si on connaît la surface des rizières cultivées sur le bassin versant terrestre du Vaccarès, on peut estimer le volume global des eaux rizicoles drainées par le Vaccarès pendant une campagne culturale, sans toutefois pouvoir préciser la répartition de cette eau au cours de cette campagne. $\mathrm{Sr} \mathrm{Fu}, \mathrm{Sr}$ Ro et Sr Riv étant les surfaces en hectares cultivées en rizières sur les bassins de Fumemorte, de Roquemaure et sur les propriétés riveraines, ce volume en millions de $\mathrm{m}^{3}$ est :

$\mathrm{V}=10000(\mathrm{Sr} \mathrm{Fu}+\mathrm{Sr} \mathrm{Ro})+12000 \mathrm{Sr} \mathrm{Riv}+5^{*}$ (* apport du bassin nord via le canal de Roquemaure).

\subsection{L'évacuation du trop-plein des étangs à la mer}

L'ouverture et la fermeture des vannes des pertuis de la Digue à la mer sont assurées manuellement par le garde-digue en fonction de la dénivellation entre la mer et les étangs et de la politique définie par l'organisme de gestion de la digue. Cette particularité et les caractéristiques techniques des vannages se prêtent mal au calcul précis des volumes débités. Ces volumes ne peuvent faire l'objet que d'estimations. Au début đẹs années 1950, la Direction. départementale de l'agriculture considérait que les ouvrages de la Digue à la mer permettaient d'évacuer environ le dixième de l'apport total d'eau du Rhône à la Camargue, soit 40 à 50 millions de $\mathrm{m}^{3}$ par an. Pour ma part, j'ai tenté d'établir, à l'échelle du mois, une relation simple entre le niveau moyen des étangs et le volume potentiel évacué. Pour cela j'ai analysé les enregistrements de niveaux d'eaux effectués de part et d'autre des pertuis depuis 1968 et $\mathrm{j}$ 'ai tiré parti de fréquentes observations du fonctionnement des vannages en fonction de leur état, pour différents niveaux des étangs et dans toutes les situations climatiques possibles. Pour imprécise que soit cette méthode, les résultats qu'elle donne sont révélateurs de l'importance d'une bonne gestion des vannages de la digue pour réguler les niveaux d'eau et la salinité des étangs. D'après mes calculs, au cours des quatre décennies passées, ces vannages auraient permis l'évacuation de quelque $950 \mathrm{Mm}^{3}$ d'eau représentant une perte de sels d'environ 10 millions de tonnes (Tableau 2).

\subsection{Les introductions d'eau de mer, volontaires ou accidentelles}

Les entrées d'eau de mer, occultes ou clandesti= nes, ne sont pas chiffrables directement. Elles sont déduites des augmentations du stock de sel du système d'étangs (Heurteaux 1992). D'importantes manipulations illicites des vannes de la Digue à la mer ont eu lieu de 1978 à 1988, surtout en été et en automne (Tableau 3). En outre, au cours de la décennie 1980, le mauvais état de la Digue à la Mer a été favorable aux intrusions marines insidieuses ou brutales, estimées à quelque 20 millions de $\mathrm{m}^{3}$ dont 7 en 1982 et 5 en 1985.

\section{Exemples de calcul de bilans hydriques du système Vaccarès}

\section{5:1. Les données mesurées et leur utilisation}

Les seules données mesurées, donc connues avec une assez bonne précision, sont les niveaux et la salinité des étangs, la pluie et les autres grandeurs météorologiques qui permettent de calculer l'évaporation.

1) Les variations du niveau de l'étang du Vaccarès sont enregistrées à l'échielle de Fumemorte (n Fu). A partir des niveaux du Vaccarès, on calcule : 
Tableau 2. Estimations trimestrielles du volume moyen, en millions de $\mathbf{m}^{3}$, du trop-plein de étangs, évacué par les vannes de la Digue à la Mer.

Table 2. Three-monthly estimates of the mean volume of excess water from the lagoons evacuated by the sluices in the sea wall, in millions de $\mathrm{m}^{3}$.

\begin{tabular}{|c|c|c|c|c|c|c|c|c|c|c|}
\hline \multicolumn{3}{|c|}{ Décennie 1950} & \multicolumn{3}{|c|}{ Total décennie: } & \multicolumn{2}{|c|}{$335 \mathrm{Mm} 3$} & \multirow[b]{2}{*}{1958} & \multirow[b]{2}{*}{1959} & \multirow[b]{2}{*}{1960} \\
\hline période & 1951 & 1952 & 1953 & 1954 & 1955 & 1956 & 1957 & & & \\
\hline ler trimestre & 7 & 36 & 0 & 28 & 35 & 11 & 6 & 5 & 4 & 28 \\
\hline 2ème trimestre & 10 & 4 & 0 & 7 & 4 & 5 & 2 & 0 & o & 6 \\
\hline 3ème trimestre & 3 & 0 & 3 & $\mathbf{0}$ & 0 & 0 & $\mathbf{0}$ & 0 & 0 & 0 \\
\hline 4ème trimestre & 45 & 0 & 57 & $\mathbf{0}$ & 6 & 4 & 2 & 0 & 11 & 6 \\
\hline année & 65 & 40 & 60 & 35 & 45 & 20 & 10 & 5 & 15 & 40 \\
\hline \multicolumn{3}{|c|}{ Décennie 1960} & \multicolumn{3}{|c|}{ Total décennie : } & \multicolumn{2}{|c|}{$170 \mathrm{Mm} 3$} & & & \\
\hline période & 1961 & 1962 & 1963 & 1964 & 1965 & 1966 & 1967 & 1968 & 1969 & 1970 \\
\hline ler trimestre & 17 & 7 & 5 & 21 & 4 & 9 & 0 & 0 & 11 & 27 \\
\hline 2ème trimestre & 0 & 0 & 1 & 6 & 0 & 0 & 0 & 0 & 12 & 1 \\
\hline 3ème trimestre & 0 & 0 & 0 & 0 & 0 & 0 & 0 & 0 & 0 & 0 \\
\hline 4ème trimestre & 4 & 0 & 12 & 8 & 1 & 1 & 0 & 0 & 23 & 0 \\
\hline année & 21 & 7 & 18 & 35 & 5 & 10 & 0 & 0 & 46 & 28 \\
\hline \multicolumn{3}{|c|}{ Decennie 1970} & \multicolumn{3}{|c|}{ Total décennie : } & \multicolumn{2}{|c|}{$295 \mathrm{Mm} 3$} & & & \\
\hline période & 1971 & 1972 & 1973 & 1974 & 1975 & 1976 & 1977 & 1978 & 1979 & 1980 \\
\hline ler trimestre & 2 & 24 & 17 & 26 & 0 & 10 & 31 & 48 & 11 & 13 \\
\hline 2ème trimestre & 0 & 11 & 1 & 14 & 0 & 3 & 9 & 12 & 3 & 2 \\
\hline 3ème trimestre & $\mathbf{0}$ & o & 0 & 0 & $\mathbf{0}$ & 0 & 3 & 2 & 0 & 0 \\
\hline 4ème trimestre & 0 & 2 & 2 & 0 & 0 & 14 & 28 & 0 & 7 & 0 \\
\hline anné & 2 & 37 & 20 & 40 & 0 & 27 & 71 & 62 & 21 & 15 \\
\hline \multicolumn{3}{|c|}{ Décennie 1980} & \multicolumn{3}{|c|}{ Total décennie : } & \multicolumn{2}{|c|}{$150 \mathrm{Mm} 3$} & & & \\
\hline période & 1981 & 1982 & 1983 & 1984 & 1985 & 1986 & 1987 & 1988 & 1989 & 1990 \\
\hline 1er trimestre & 0 & 0 & $\overline{6}$ & 0 & 8 & 21 & 23 & 36 & 0 & 0 \\
\hline 2ème trimestre & 0 & 0 & 4 & 0 & 5 & 7 & 7 & 7. & 0 & 0 \\
\hline 3ème trimestre & $\mathbf{0}$ & $\mathbf{0}$ & $\mathbf{0}$ & 0 & $\mathbf{0}$ & o & 0 & 0 & 0 & 0 \\
\hline 4ème trimestre & 0 & 2 & 0 & 4 & 0 & 6 & 3 & 0 & 0 & 11 \\
\hline année & 0 & 2 & 10 & 4 & 13 & 34 & 33 & 43 & 0 & 11 \\
\hline
\end{tabular}

Tableau 3. Estimation des introductions volontaires d'eau de mer, en millions de $\mathbf{m}^{3}$, de 197 à 1988 .

Table 3. Estimated intentional inputs of sea water from 1978 to 1988 , in millions $\mathrm{m}^{3}$.

\begin{tabular}{cccccccccccc}
\hline 1978 & 1979 & 1980 & 1981 & 1982 & 1983 & 1984 & 1985 & 1986 & 1987 & 1988 & total \\
\hline 4 & 8 & 1 & 7 & 6 & 2 & 3 & 8 & 5 & 5 & 1 & 50 \\
\hline
\end{tabular}

- le volume de chaque étang pour lesquels il existe des cartes bathymétriques, (Parc naturel régional de Camargue, 1986 ; Heurteaux.1992) ; on en tire les formules ci-dessous permettant de calculer directement, en millions de $\mathrm{m}^{3}$ à $2 \%$ près, le volume total du système d'étangs

$n$ Fu compris entre $-0,20$ et $+0,20 \mathrm{~m} \mathrm{NGF,} \mathrm{V}=1,16 \cdot \mathrm{Fu}+114$

$\mathrm{n} \mathrm{Fu}<-0,20 \mathrm{~m} \mathrm{NGF}$, $\mathrm{V}=1,05 \mathrm{Fu}+111$

$\mathrm{n} \mathrm{Fu}>+0,20 \mathrm{~m} \mathrm{NGF}$, $\mathrm{V}:=1,28 \mathrm{Fu}+112$ 
- les cofficients à appliquer pour quantifier les apports pluviaux aux Etangs inférieurs et les pertes par évaporation des différents plans d'eau (Tableau 1).

2) Le dêtail des épisodes pluvieux aux six stations mentionnées dans les tableaux climatologiques de Météofrance permettent d'estimer l'état hydrique du bassin versant terrestre et de calculer les apports pluviaux aux étangs.

3) Les valeurs mensuelles de l'évaporation sont calculées par la méthode de Penman.

4) La salinité des étangs, permet de corriger l'évaporation Penman.

A partir de ces données, pour le pas de temps choisi, on calcule :

- d'une part, le volume initial et le volume final des étangs et, par différence des deux, le stockage ou le destockage d'eau ;

- d'autre part, le volume apporté par les pluies et le volume soustrait par évaporation ; ayant, par ailleurs, une estimation du volume des colatures agricoles drainées au Vaccarès, des éventuelles vidanges du trop plein des étangs et des éventuelles entrées d'eau de mer par la Digue à la mer, il est possible d'établir le bilan des entrées et des sorties d'eau du système Vaccarès et de les comparer aux variations de volumes calculées d'après les variations de niveaux.

Dans l'état actuel de nos connaissances, compte tenu des approximations faites, il paraît raisonnable de choisir un pas de temps large. Dans l'année, deux périodes de six mois présentent des caractéristiques hydriques très différentes :

- la période des cultures, d'avril à fin septembre, au cours de laquelle le bilan des pluies et de l'évaporation est globalement déficitaire alors que les introductions d'eau du Rhône représentent quelque $95 \%$ du total annuel (Heurteaux et al. 1992, Heurteaux 1993).

- la période hors-cultures, d'octobre à fin mars, où le bilan des pluies et de l'évaporation est globalement excédentaire et l'irrigation inexistante.

5.2. Exémple de bilan hydrique calculé pour la période du $1^{\text {er }}$ octobre 1984 au 30 septembre 1986 (Tableau 4).

\section{Conclusion}

Le calcul à l'échelle de l'année de l'apport pluvial aux étangs et des départs par évaporation en fonction des lames d'eau précipitées ou évaporées et des niveaux d'eau (Tableau 5) révèle les tendances moyennes suivantes:

1) Le ruissellement sur le bassin versant terrestre du Vaccarès réduit à $130 \mathrm{~km}^{2}$, établi en fonction des critères retenus, fournit sensiblement autant d'eau à cet étang que l'interception directe, sauf dans les cas extrêmes, les fortes sécheresses accentuant la capacité de rétention des terrains et les pluies diluviennes ayant l'affet inverse. Le ruissellement pluvial représente plus de $50 \%$ de la pluie incidente (cœfficient de ruissellement $=0,52$; écart-type 0,12 ; extrêmes $0,22(1989)-0,64$ (1977).

2) Le rendement des plutes ramené au $\mathrm{km}^{2}$ de bassin versant total est peu différent pour le Vaccarès $\left(0,47 \mathrm{Mm}^{3} / \mathrm{km}^{2}\right)$ et pour les Etangs inférieurs $(0,50$ $\mathrm{Mm}^{3} / \mathrm{km}^{2}$ ). Par contre, ramené au $\cdot \mathrm{km}^{2}$ de surface en eau, le rendement des pluies est presque deux fois plus grand pour le Vaccarès $\left(1,41 \mathrm{Mm}^{3} / \mathrm{km}^{2}\right)$ que pour les Etangs inférieurs $\left(0,73 \mathrm{Mm}^{3} / \mathrm{km}^{2}\right)$.

3) Les volumes évaporés sont relativement stables d'une année à l'autre, surtout au niveau des Etangs inférieurs où, au cours des années sèches, l'augmentation de la lame d'eau évaporée compense la diminution de la surface des plans d'eau, sauf si celle-ci est trop importante, comme en 1989.

4) Le bilan de l'apport pluvial et de l'évaporation est toujours déficitaire au niveau des Etangs inférieurs, même au cours d'années très pluvieuses, alors qu'il est le plus souvent excédentaire pour le Vaccarès. Il y a donc une tendance naturelle à l'écoulement du Vaccarès vers les Etangs inférieurs.

5) Pour l'ensemble du système, l'apport pluvial annuel moyen $\left(124 \mathrm{Mm}^{3}\right)$ et le volume annuel moyen soustrait par évaporation $\left(130 \mathrm{Mm}^{3}\right)$ sont proches de l'équilibre avec une légère tendance au déficit. Les années sèches ou au contraire humides sont marquées par un déficit ou un excédent hydrique d'une quarantaine de millions de $\mathrm{m}^{3}$. L'apport d'eau de drainage agricole (actuellement 30 a 40 millions de $\mathrm{m}^{3}$ par an) compense peu ou prou le déficit hydrique mais aggrave l'excédent. Dans ce cas, la Camargue n'échappe à l'inondation généralisée par débordement des étangs, que par l'accroissement des sorties d'eau par les vannages de la Digue à la Mer. 
Tableau 4. Bilan hydrique du système Vaccarès du $1^{\text {er }}$ octobre 1984 au 30 septembre 1986.

Table 4. Hydrological balance for the Vaccarès system from 1 October 1984 to 30 September 1986.

\begin{tabular}{ccccc}
\hline termes & période & période & période & période \\
du & du $1-10-84$ & du $1-04-85$ & du $1-10-85$ & du $1-04-86$ \\
bilan & au 31-03-85 & au 30-09-85 & au 31-03-86 & au 30-09-86 \\
\hline
\end{tabular}

A-Variations du volume des étangs calculées à partir des niveaux du Vaccarès niveau initial ni

\begin{tabular}{|c|c|c|c|c|}
\hline $\begin{array}{l}\text { m NGF } \\
\text { volume initial } \mathrm{Vi}\end{array}$ & $-0,26$ & $+0,13$ & $-0,13$ & $+0,20$ \\
\hline $\begin{array}{l}\mathrm{Mm3} \\
\text { niveau final ni }\end{array}$ & 84 & 129 & 98 & 137 \\
\hline $\begin{array}{l}\text { m NGF } \\
\text { volume final } \cdot v f\end{array}$ & $+0,13$ & $-0,13$ & $+0,20$ & $+0,02$ \\
\hline $\mathrm{Mm} 3$ & 129 & 98 & 137 & 116 \\
\hline $\mathrm{Vf}-\mathrm{Vi} \mathrm{Mm} 3$ & +45 & -31 & +39 & -21 \\
\hline
\end{tabular}

B-Bilan des entrées et des sorties d'eau

\begin{tabular}{lcccc} 
ENTREES Mm3 & & & & \\
pluie & 77 & 26 & 79 & 37 \\
drainage agricole * & 2 & 26 & 2 & 28 \\
bassin nord & 2 & 6 & 3 & 6 \\
mer & 6 & 8 & 8 & 8 \\
eaux souterraines & $?$ & + & 1 & $?$ \\
\cline { 2 - 5 }$\quad$ total Mm3 & 87 & 66 & 93 & 79 \\
\hline
\end{tabular}

\begin{tabular}{lcccc} 
SORTIES Mm3 & & & & \\
Évaporation & 31 & 100 & 30 & 101 \\
Digue à la Mer & 9 & 0 & 21 & 7 \\
\cline { 2 - 5 }$\quad$ & 40 & 100 & 51 & 108 \\
\cline { 2 - 5 } & & & & \\
\cline { 2 - 5 } Sotal $\mathrm{Mm} 3$ & $\mathrm{Mm} 3 * *$ & -34 & $+42-$ & -29 \\
\hline
\end{tabular}

* Le drainage agricole provient des rizières en période culturale

et des vignes hors période culturale

** Le solde des entrées et des sorties d'eau est en principe égal à vf - vi

Les approximations qu'il faut faire pour quantifier les termes du bilan hydrique du système Vaccarès sont révélatrices des insuffisances du réseau hydrométrique de la Camargue, du manque de rationalisme dans la gestion des différents ouvrages hydrauliques, de l'absence de coordination entre les différents services ayant cette gestion en charge et, enfin, de l'importance des lacunes dans la connaissance des phénomènes hydrodynamiques souterrains ou de surface qui régissent le fonctionnement hydrauliques de la Camargue.
Comme c'est le cas pour toutes les zones humides où la pression humaine est forte, la sauvegarde de la Camargue passe par une gestion programmée de l'eau. Une telle programmation est également nécessaire à une cohabitation harmonieuse des différents intérêts économiques. Or, le système des étangs centraux régule le fonctionnement hydraulique de toute l'Ile de la Camargue. L'établissement d'un modèle prédictif de gestion de l'hydraulique de ce territoire requiert donc pour être fiable une quantification des différents termes du bilan hydrique 
Tableau 5. Evolution des volumes annuels, en millions de $\mathrm{m}^{3}$, apportés aux étangs du système Vaccarès par les pluies et soustraits par évaporation en fonction de l'importance de la surface occupée par les plans d'eau, des précipitations et de l'évaporation, pour dix valeurs de celles-ci réparties entre les extrêmes mesurés.

Table 5. Changes in the annual inflows of rain water into the lagoons of the Vaccarès system and the annual volume evaporating from the lagoons (in millions of cubic metres), according to water surface area and ten amounts of rainfall and evaporation distributed between their two recorded extreme values.

\begin{tabular}{|c|c|c|c|c|c|}
\hline & $\begin{array}{c}\text { pluie sur BV } \\
\text { hauteur en mètres } \\
\text { et années }\end{array}$ & $\begin{array}{l}\text { apport pluvial } \\
\text { du BV terrestre } \\
\text { du Vaccarès }\end{array}$ & $\begin{array}{l}\text { apport pluvial } \\
\text { direct } \\
\text { au Vaccarès }\end{array}$ & $\begin{array}{l}\text { apport pluvial } \\
\text { aux Etangs } \\
\text { inférieurs }\end{array}$ & $\begin{array}{c}\text { apport pluvial } \\
\text { total au } \\
\text { système d'étangs }\end{array}$ \\
\hline & $0,24(1989)$ & 7 & 16 & 6 & 29 \\
\hline & $0,47(1985)$ & 30 & 32 & 20 & 82 \\
\hline & $0,47(1988)$ & 37 & 34 & 23 & 94 \\
\hline & $0,61 \quad(1987)$ & 41 & 41 & 32 & 114 \\
\hline & $0,70(1975)$ & 44 & 45 & 31 & 120 \\
\hline & $0,74(1986)$ & 50 & 50 & 40 & 140 \\
\hline & $0,75(1990)$ & 48 & 50 & 34 & 132 \\
\hline & $0,86(1977)$ & 72 & 59 & 47 & 178 \\
\hline & $0,90(1976)$ & 69 & 59 & 43 & 171 \\
\hline & $0,91(1972)$ & 70 & 60 & 51 & 181 \\
\hline moy. & 0,665 & 46,8 & 44,6 & 32,7 & 124,1 \\
\hline & $\begin{array}{c}\text { Evaporation } \\
\text { en mètres avec } \\
\text { correction de salinité }\end{array}$ & $\begin{array}{c}\text { volume évaporé } \\
\text { sur le } \\
\text { Vaccarès }\end{array}$ & $\begin{array}{l}\text { volume évaporé } \\
\text { sur les } \\
\text { Etangs inférieurs }\end{array}$ & $\begin{array}{l}\text { volume total } \\
\text { évaporé sur le } \\
\text { système d'étangs }\end{array}$ & \\
\hline & $1,08(1987)$ & 72 & 53 & 125 & \\
\hline & $1,09(1977)$ & 73 & 54 & 127 & \\
\hline & $1,11(1972)$ & 74 & 55 & 129 & $\because$ \\
\hline & $1,11(1986)$ & 74 & 55 & 129 & \\
\hline & $1,14(1985)$ & 76 & 54 & 130 & \\
\hline & $1,17(1989)$ & 79 & 45 & 124 & \\
\hline & $1,18(1988)$ & 78 & 54 & 132 & \\
\hline & $1,19(1975)$ & 79 & 54 & 133 & \\
\hline & $1,20(1990)$ & 79 & 54 & 133 & \\
\hline & $1,24 \cdot(1976)$ & 82 & 56 & 138 & \\
\hline moy. & $\quad 1,151$ & 76,6 & 53,4 & 130 & \\
\hline
\end{tabular}

des étangs plus précise que celle à laquelle nous aboutissons actuellement. Pour cela, un approfondissement de nos connaissances sur l'hydrodynamique des étangs du système Vaccarès s'impose.

\section{Travaux cités}

Godin L: 1990. - Impact de l'irrigation pour la riziculture sur l'hydrologie et la chime des eaux en Camargue. Approche à partir du bassin d'irrigation de l'Aube de Bouic. Thèse Doct. Univers. Franche Comté, 172 p.
Heurteaux P. 1988. - La dynamique de l'eau et du sel des étangs du système Vaccarès. Rapport G.I.S. polycop. en 5 parties. Parc naturel régional de Camargue (éd,). lère partie : L'évolution du niveau du Vaccarès depuis 1951,34 p. 2ème partie : Essai de quantification des termes du bilan hydrique des étangs du système Vaccarès, 43 p. 3ème partie : La circulation des eaux entre les différents étangs du système Vaccarès. Phénomènes inducteurs et conséquences, $47 \mathrm{p}$. 4ème partie : L'évolution du stock de sels dissous dans les étangs du système Vaccarès au cours des quarante dernières années, 34 p. Sème partie : Les mécanismes inducteurs des pertes et des gains de sels par les étangs du système Vaccarès, $26 \mathrm{p}$. 
Heurteaux P. 1990. - La formule de Penman et la quantification de l'évaporation et de l'évapotranspiration en Camargue, notamment de l'évapotranspiration des rizières. Centre français du riz. Doc. polyc., $51 \mathrm{p}$.

Heurteaux P. 1992. - Modifications du régime hydrique et salin des étangs du système Vaccarès (Camargue, France) liées aux perturbations anthropiques des cinquante dernières années. Annls limnol. 28 (2) : 157-174.

Heurteaux P. 1994. - La Camargue et le Rhône. Les usages de l'eau du fleuve. Courrier du Parc naturel régional de Camargue $n^{\circ} 41$.

Heurteaux P., Crombé O. \& Toni Cl. 1992. - Essai de quantification de l'eau d'irrigation introduite en Grande Camargue, notamment pour la riziculture. Ecologia mediterranea $X V I I I: 31-48$.

Ministère de l'Agriculture (éd.). 1970. - Camargue. Etude hydrogéologique, pédologique et de salinité. 4 volumes en coffret, $372 p$ + annexes + cartes.

Ministère de l'Agriculture (éd.). Direction départementale de l'agriculture des Bouches-du-Rhône. 1975. - Détermination du cycle annuel normal du niveau de l'étang du Vaccarès. Camargue, études hydrauliques et hydrologiques. Doc. polyc., $23 \mathrm{p}+$ annexes.
Ministère de l'Agriculture. 1975. - La maîtrise des plans d'eau en Camargue. Livre blanc. DDAF, $70 \mathrm{p}$.

Ministère de l'Agriculture. 1975. - Les groupements hydrauliques agricoles des Bouches-du-Rhồne. Assainissement Camargue. DDAF Marseille-Arles. Service hydraulique, $45 \mathrm{p}$.

Ministère de l'Agriculture. 1983. - Les groupements hydrauliques agricoles des Bouches-du-Rhône. Irrigationassainissement. Les stations de pompage collectives de l'arrondissement d'Arles. DDAF Marseille-Arles. Service hydraulique, $106 \mathrm{p}$.

Parc naturel régional de Camargue (éd.). 1986. - Bathymétrie du Vaccarès et des Etangs inférieurs. Doc, polycop. \& cartes. Réserve naturelle et SNPN .. Gis Camargue, $12 \mathrm{p}$.

Parc naturel régional de Camargue. 1992. - Occupation du sol en 1991 et évolution depuis 1970. Agence régionale pour.l'environnement PACA, $31 \mathrm{p}+$ cartes.

Vaquer A. \& Heurteaux P. 1989. - Modifications récentes de la végétation aquatique de l'étang du Vaccarès (Camargue, France) liées aux perturbations anthropiques. Annis Limnol. 25 (1) : 25-38. 